30TH INTERNATIONAL COSMIC RAY CONFERENCE

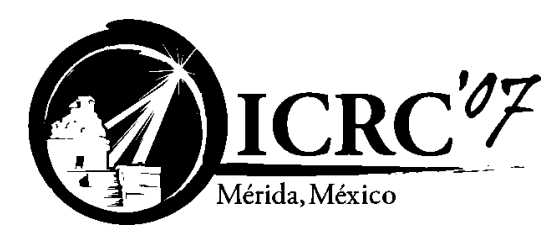

\title{
Detection of Cherenkov light from air showers with Geiger-APDs
}

\author{
A. N. Otte ${ }^{1,2}$, I. Britvitch ${ }^{3}$, A. Biland ${ }^{3}$, F. Goebel ${ }^{1}$, E. Lorenz ${ }^{3}$, F. Pauss ${ }^{3}$, D. Renker ${ }^{4}$, U. \\ RÖSER $^{3}$, T. SCHWEIZER ${ }^{1}$ \\ ${ }^{1}$ Max-Planck-Institut für Physik, D-80805 München, Germany \\ ${ }^{2}$ Humboldt-Universität zu Berlin, D-12489 Berlin, Germany \\ ${ }^{3}$ ETH Zurich, CH-8093 Zurich, Switzerland \\ ${ }^{4}$ Paul Scherrer Institut, CH-5232 Villigen, Switzerland \\ otte@mppmu.mpg.de
}

\begin{abstract}
We have detected Cherenkov light from air showers with Geiger-mode APDs (G-APDs). GAPDs are novel semiconductor photon-detectors, which offer several advantages compared to conventional photomultiplier tubes in the field of ground-based $\gamma$-ray astronomy. In a field test with the MAGIC telescope we have tested the efficiency of a G-APD / light catcher setup to detect Cherenkov light from air showers. We estimate a detection efficiency, which is $60 \%$ higher than the efficiency of a MAGIC camera pixel. Ambient temperature dark count rates of the tested G-APDs are below the rates of the night sky light background. According to these recent tests G-APDs promise a major progress in ground-based gamma-ray astronomy.
\end{abstract}

\section{Introduction and Motivation}

Ground-based $\gamma$-ray astronomy is a rapidly expanding and successful field in astroparticle physics. The Very High Energy (VHE) $\gamma$-ray astronomy window was opened by the Whipple collaboration with the detection of the Crab Nebula at energies above $1 \mathrm{TeV}$ using the imaging air Cherenkov telescope (IACT) technique [1]. An IACT records by means of a finely segmented PMT camera the very weak Cherenkov light flashes from VHE $\gamma$-ray induced air showers. Since 1989 more than 70 sources of VHE- $\gamma$-rays have been detected. Most of them have been discovered in the last four years by the most recent generation of IACTs.

The MAGIC telescope is an IACT with a $17 \mathrm{~m}$ diameter reflector. It is currently the largest and technologically most advanced IACT. MAGIC is aiming to uncover the so far nearly unexplored $\operatorname{VHE} \gamma$ ray domain between 30 and $150 \mathrm{GeV}$, important for the study of several fundamental physics questions such as the extragalactic background light, gamma ray bursts, pulsars, dark matter and tests of quantum gravity.
Increasing the sensitivity and/or lowering the threshold in this important energy region requires telescopes with a higher collection efficiency for Cherenkov photons. While it seems difficult and expensive to increase the reflector area much beyond the area of existing telescopes, it is more promising to develop photon detectors with higher photon detection efficiencies (PDE).

Since a few years, a new type of semiconductor photon detector is being developed with single photon resolution and a potential for much higher PDEs than that of PMTs. The so-called G-APD is now in the transition from an R\&D device to a commercial product. The size of some G-APDs is now sufficiently large to evaluate their possible use in IACTs.

We carried out some first tests, which showed that Cherenkov light from air showers can be detected with latest G-APDS [2]. After these promising tests we have placed a matrix of four G-APDs in the focal plane of the MAGIC telescope. The aim of this test was to compare the G-APD to a MAGIC camera pixel in terms of their efficiency to detect Cherenkov light. The results of this field test are presented here. 


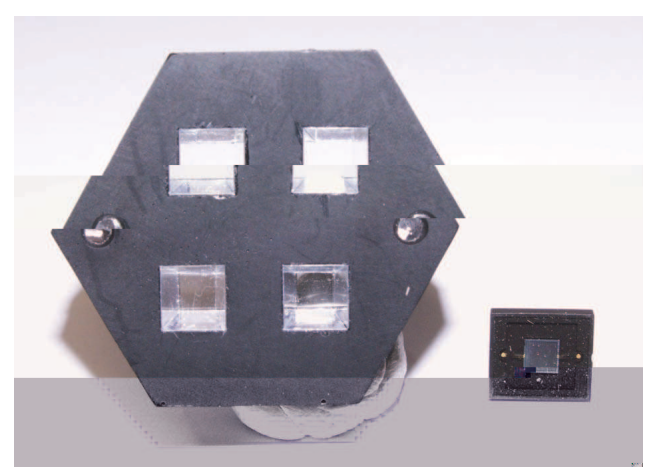

Figure 1: Front view of the matrix of four light catchers with entrance areas of $6 \times 6 \mathrm{~mm}^{2}$ and exit areas of $3 \times 3 \mathrm{~mm}^{2}$. One of the tested $3 \times 3 \mathrm{~mm}^{2}$ MPPCs is shown in the lower right side. In the test, this MPPC was coupled to the exit area of one of the light catchers.

\section{The G-APD}

The G-APD or SiPM, MPPC, MRS-APD, ... was initially developed by several Russian groups [3, 4, 5]. In a G-APD typically 100 to 1000 small avalanche photo diodes (cells) are integrated per $\mathrm{mm}^{2}$ detector area. Each cell operates autonomous in limited Geiger mode. The output of a G-APD is the sum signal of all cells.

The main advantage of G-APDs employed in astroparticle physics is the potential high PDE, which can be up to a factor of three higher than that of classical PMTs. Other advantages are:

- single photoelectron response

- compactness

- insensitivity to magnetic fields

- low operation voltage $(<100 \mathrm{~V})$ and high gains $\left(10^{5}-10^{6}\right)$

- no damage when exposed to sunlight, even when under bias

- long-term prospects for low fabrication costs

Disadvantages of G-APDs are:

- current sensors sizes are limited to $<10 \mathrm{~mm}^{2}$
- dark noise rates between $100 \mathrm{kHz}$ and several $\mathrm{MHz}$ per $\mathrm{mm}^{2}$ sensor area at room temperature

- so-called optical crosstalk, i.e. correlated firing of several cells

For our tests we have used four $3 \times 3 \mathrm{~mm}^{2}$ prototype G-APDs from Hamamatsu; MPPCs with about $50 \%$ peak PDE in the blue spectral range, cell sizes of $50 \times 50 \mu \mathrm{m}^{2}, \sim 2 \mathrm{MHz}$ dark rate at room temperature, and signal rise- and fall-times of $4 \mathrm{~ns}$ and $100 \mathrm{~ns}$ respectively.

\section{Detection of Cherenkov light using the MAGIC telescope}

For the detection of Cherenkov light using the MAGIC telescope we grouped the aforementioned 4 MPPCs in a $2 \times 2$ matrix (s. Figure 1 ). The effective area of each sensor was enhanced by a compound miniature light concentrator. The first stage is a light catcher made of a high reflective foil (95\% reflectivity for wavelengths down to $320 \mathrm{~nm}$ ). The second stage is a light catcher made of plexiglas.

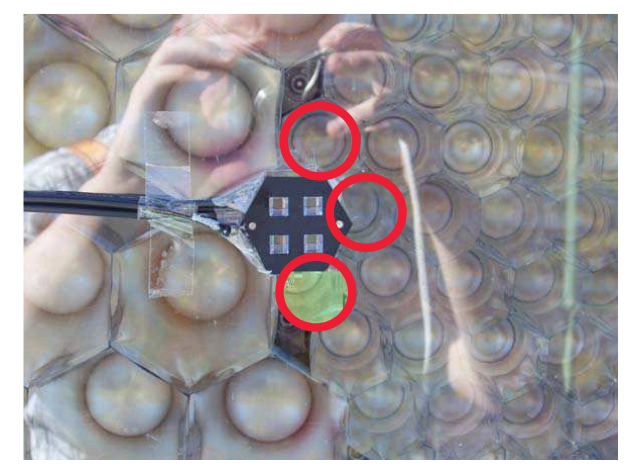

Figure 2: MPPC matrix mounted onto the entrance window of the MAGIC camera. The three red circles mark the PMTs, whose signals the MPPC signals were compared to.

The MPPC-matrix was mounted onto the entrance window of the MAGIC camera lined up with one pixel in the outermost ring of the finer pixelated inner camera (s. Figure 2). The MPPC signals were amplified with MAR 8-ASM amplifiers from Mini-Circuits. After the amplifier the signals were 

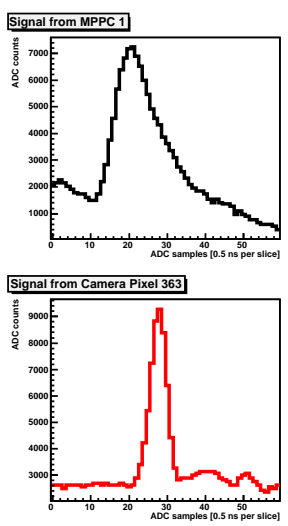
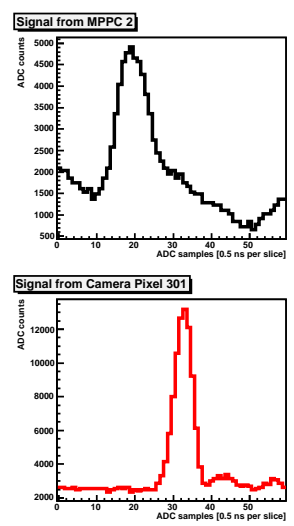
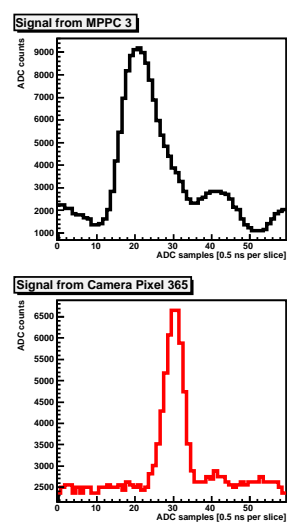
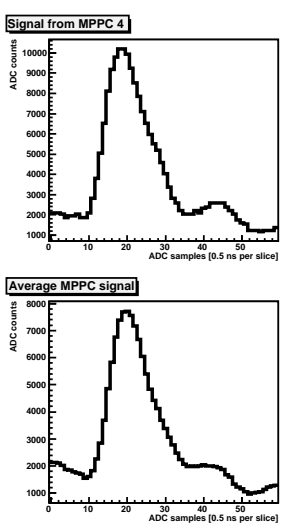

Figure 3: The upper row shows the signals of the four MPPCs for the event shown in Figure 4. The average over the four MPPCs is shown in the rightmost figure in the lower row. The three recorded signals shown in the lower row in red are from the three PMTs surrounding the MPPC array.

clipped with a $2 \mathrm{~ns}$ long cable to avoid the $>100 \mathrm{~ns}$ long tails of the MPPC signals, i.e., to minimize baseline shifts and pile-up. The analog signals were then sent via $160 \mathrm{~m}$ long optical links into the counting house were they were digitized by the MAGIC-DAQ [6] at a sampling frequency of 2 GSamples/s each time the readout was triggered by a cosmic ray shower.

In this configuration each MPPC recorded a single photon rate from the light of night sky of about $25 \mathrm{MHz}$. This rate is about 10 times higher than the intrinsic dark rate of the MPPC at room temperature. Thus in future IACT applications of G-APDs, only moderate requirements are needed in terms of temperature stabilization and cooling.

Figure 4 shows one image of an air shower that was recorded during the test. For this event clear signals are seen by all four MPPCs (see upper row of Figure 3). The signals recorded by the three PMTs closest to the MPPC-array (red circles in Figure 2) are shown by the three red graphs in the lower row of the Figure 3.

In the above event each MPPC recorded on average 12 photoelectrons and the surrounding PMTs on average 130 photoelectrons. Note that the entrance area of the light catcher of a PMT and a MPPC differ by a factor 20. Therefore, the PMT signals have to be scaled down by this factor in order to allow for a quantitative comparison between both

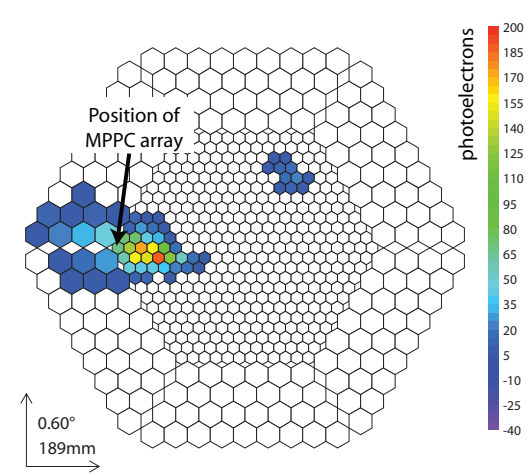

Figure 4: One event recorded during the test. The corresponding signals of the MPPCs and of the three PMTs surrounding the MPPC array are shown in Figure 3. Mark position of the MPPC array.

sensors, i.e. $130 / 20 \sim 6$ photoelectrons, which is half the intensity recorded by the MPPCs.

For about 300 events we calculated the photoelectron intensity recorded by the MPPCs and the area scaled intensity recorded by the surrounding PMTs. Figure 5 shows the correlation between the MPPC and PMT recorded intensities. In the figure the number of photoelectrons recorded by the MPPCs is above the dashed line through the origin 


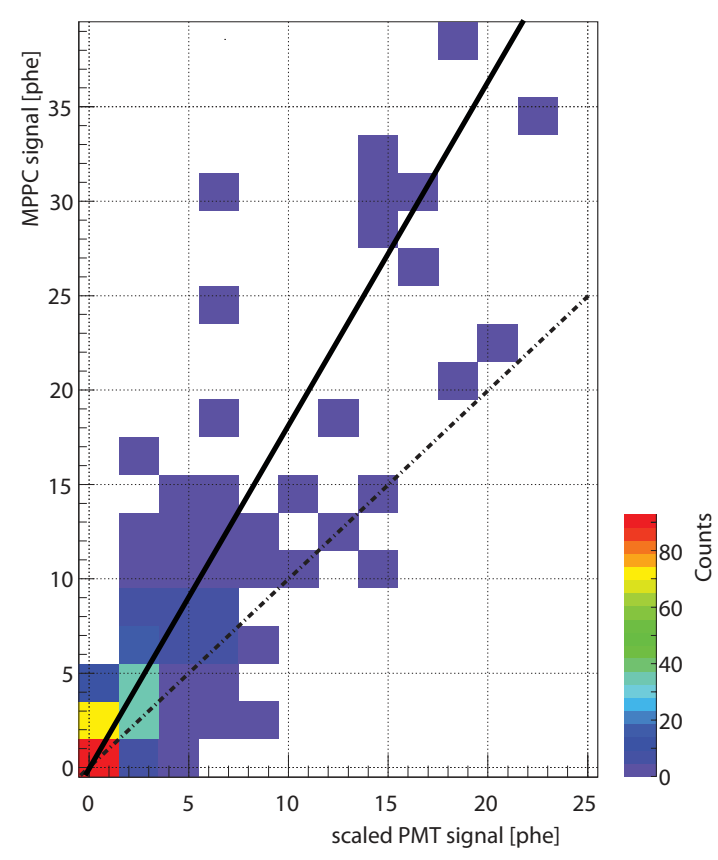

Figure 5: Comparison of the MPPC and PMT photoelectron signal scaled to the MPPC area.

(slope 1) for most of the events, which indicates that the MPPCs have a higher PDE than the PMTs. On average the MPPCs record a $80 \%$ higher photoelectron intensity than the PMTs, indicated by the solid line in Figure 5 (slope 1.8). However, this value is not corrected for optical crosstalk, which results in an overestimate of the PDE of the MPPCs. After correcting for optical crosstalk we estimate that the MPPC light catcher arrangement is $60 \%$ more sensitivity than the PMT light catcher arrangement used in MAGIC.

Note that above we not only estimated the PDE of the MPPC. Instead the combined efficiency of the photondetector and the light catcher was estimated. The MPPC light catcher was not optimized, which leaves room for further improvement.

\section{Discussion and Conclusion}

In summary we can conclude the following results:
- It is possible to detect Cherenkov light from air showers with available G-APDs

- With the tested G-APDs, we obtained a significant increase in the efficiency to collect Cherenkov photons from air showers compared to a MAGIC PMT-pixel ( $\approx 60 \%)$

- The intrinsic noise rate of the tested G-APDs at room temperature was a factor of 10 below the level of the night sky background light

As a consequence of the significant increase in PDE we conclude that by means of G-APDs one can either increase the sensitivity and lower the threshold of IACTs or one can reduce the reflector area of telescopes while conserving the same performance in case classical PMTs are used.

Even if available G-APD already show impressive characteristics further improvements are desirable like larger sensor areas of $5 \times 5 \mathrm{~mm}^{2}$ up to $10 \times 10 \mathrm{~mm}^{2}$, a reduction of optical crosstalk to well below $5 \%$, and a further reduction of the intrinsic noise to $<100 \mathrm{kHz} / \mathrm{mm}^{2}$.

\section{References}

[1] Weekes et al. ApJ, 342:379, 1989.

[2] I. Britvich et al. Vienna Conference on Instrumentation, 2007.

[3] P. Buzhan et al. NIM A, 504:48, 2003.

[4] D. Bisello et al. NIM A, 367:212, 1995.

[5] V. Golovin and V. Saveliev. NIM A, 518:560, 2004.

[6] F. Goebel et al. In This proceeding, 2007. 